\title{
Understanding and Exploiting the Internals of GPU Resource Allocation for Critical Systems
}

\author{
Alejandro J. Calderón ${ }^{1,3}$, Leonidas Kosmidis ${ }^{2}$, Carlos F. Nicolás ${ }^{3}$, Francisco J. Cazorla ${ }^{2}$, Peio Onaindia ${ }^{3}$ \\ ${ }^{1}$ Universitat Politècnica de Catalunya $\quad{ }^{2}$ Barcelona Supercomputing Center (BSC) $\quad{ }^{3}$ Ikerlan Technology Research Centre
}

\begin{abstract}
Critical real-time systems require strict resource provisioning in terms of memory and timing. The constant need for higher performance in these systems has led industry to recently include GPUs. However, GPU software ecosystems are by their nature closed source, forcing system engineers to consider them as black boxes, complicating resource provisioning. In this work we reverse engineer the internal operations of the GPU system software to increase the understanding of their observed behaviour and how resources are internally managed. This way, we allow system engineers to accurately determine the exact amount of resources required by their critical systems, avoiding underprovisioning. We first apply our methodology on a wide range of GPU hardware showing its generality in obtaining the properties of the GPU memory allocators. Next, we demonstrate the benefits of such knowledge in resource provisioning of two case studies from the automotive domain, where the actual memory consumption is up to $5.6 \times$ more than the memory requested by the application.
\end{abstract}

\section{INTRODUCTION}

In the domain of critical real-time systems we find a wide spectrum of computer systems. On the one end of the spectrum we have safety critical systems, ranging from transportation to medical and control systems. Since human lives are at stake, such systems usually have hard real-time requirements, which means that their correct behaviour is dictated not only by correct functionality but also by their timely execution with respect to predefined deadlines. On the other end we find business and mission critical systems which although do not impose a threat to human safety, their correct and timely execution is essential to fulfil their mission, typically to provide valuable services to science, society and economy. Examples of such systems are banking and commerce services, communications and scientific space missions, which have somewhat less strict timing requirements, but still important for their operation and justification of their high cost.

Despite that these systems are very diverse and have very different particular requirements, all of them have a common property: they require high availability. The key to achieve high availability is the careful resource provisioning of the system, in order to guarantee that each of the tasks of the system has enough resources to be efficiently executed, without at the same time exceeding a limit that can jeopardise the entire system or impact the other tasks.

In particular, one of the most extreme cases of resource provisioning is found in avionics [1], whose operating system standard, namely ARINC653 [2] enforces strict memory and time budgets for each task. This requires that the system engineer needs to figure out the exact memory usage of each task and ensure that the total memory usage does not exceed the size of the system memory. Similarly in timing, the worst case execution time of each task has to be determined, and ensure that it is smaller than its deadline and that the overall system has enough capacity to accommodate the execution of all tasks. Automotive operating systems, AUTOSARcompliant [3], follow a similar approach in resource allocation, as well as the operating systems in other critical domains like Integrity RTOS in industrial control systems [4].

In less critical systems built on general purpose operating systems like Unix-based ones, although the operating systems do not impose these limitations for each task, system engineers still perform the same type of analysis. For example, although these operating systems do allow the use of more memory than the one physically present in the system, based on virtual memory and disk-backed memory (a feature known as paging or swap) and/or compression, the performance of the system is severely affected when this feature is used, compromising its timing behaviour and under heavy memory pressure even the stability of the system is jeopardised. Therefore, the accurate resource provisioning allows to prevent such scenarios, guaranteeing that the total capacity of the system is not exceeded.

A recent trend in the critical domains is the introduction of GPUs, in order to satisfy the performance demand of advanced features. Probably the most well known case is in automotive, where automakers are working on autonomous driving prototype vehicles [5] powered by GPUs mainly for cognitive tasks and artificial intelligence. The medical domain and finance are also employing GPUs [6] mainly for image processing and high-computational capacity, as well as the space domain [7]. Other critical domains are expected to follow as well, especially whenever there is a need for inference based on artificial intelligence (AI) or high compute performance.

The GPU market lead vendor NVIDIA has performed significant investments in the automotive and industrial automation sector by designing embedded GPU systems meeting the temperature and reliability needs of these markets, such as the NVIDIA PX2 and its development board Jetson TX2, the NVIDIA Xavier and its latest addition NVIDIA Jetson Nano.

Despite the important performance benefits provided by GPUs, they are notoriously known about their closed source nature. In particular, NVIDIA GPUs are programmed in CUDA, a proprietary programming language developed by NVIDIA. The GPU execution model in its rudimentary form follows an accelerator approach, in which the programmer has to explicitly allocate GPU memory and manage transfers between the CPU and the GPU, as well submitting code to be executed in the GPU, known as kernel. Although this explicit resource allocation provides the delusion of full control over the resource management, the actual resource consumption both in memory and timing is larger, hidden behind closed source layers. The reason is that the actual resource management takes place within the CUDA runtime and GPU driver, which are closed source.

As a consequence, an accurate resource provisioning of GPU applications is complicated, leading either to underestimation or overestimation of resource provisioning. Although this problem is not yet very evident in the existing under-utilised prototype systems, based on Unix-like operating systems e.g. Linux, it will soon be a roadblock as these systems will require the consolidation of more software functionalities in the same platform. Even more importantly, the problem will be more pronounced when these systems will be moved to operating systems for critical systems with strict and explicit resource provisioning per task like AUTOSAR and ARINC653.

In this work, we expose for the first time the internal resource allocation mechanism of a GPU system. This way, we allow the 
accurate resource provisioning for a GPU-based critical system. First we review the different types of memory allocation in a GPU system and we start by demonstrating the basis of our methodology with a small motivational example. Next we present some essential background on memory allocators and we describe in detail our methodology to discover the properties of the memory allocator used in a GPU-based system. Finally, we present our findings for a wide range of GPUs and we use the information about the internals of the memory allocator to demonstrate the benefits of accurate resource provisioning with two case studies for a critical system, showing that the actual memory consumption is significantly higher than the one requested by the software.

\section{MEMory Allocation IN GPUs}

Before we enter into the GPU memory allocation internals, it is essential to review the programmer's view of memory management in order to better understand its internal behaviour. As already mentioned, in the CUDA programming model, the programmer is in charge of explicitly managing memory for both the CPU and the GPU side, including allocation, deallocation and transfers between the CPU and the GPU. ${ }^{1}$

Regular CPU memory ie. allocated using malloc or mmap is by default paged, which means that the operating system can swap it out to the disk if needed, typically due to memory oversubscription. On the other hand, GPU memory, allocated with cudaMalloc is always non-paged, that is, it is always present in the memory. Copies between CPU and GPU memory are performed by DMA (Direct Memory Access) operations. However, as DMA transfers are asynchronous with respect to the CPU execution, they can operate only when the pages are guaranteed to be resident in the memory. Since this is not always the case for paged memory, the transfers need to pass from a staging area of non-paged memory. In other words, in a CPU to GPU transfer, memory needs to be copied first to this intermediate buffer using the CPU and therefore synchronously, before the DMA can kick in to perform the asynchronous transfer to the device. This results in additional memory, which can be shared among applications, and additional timing overhead in GPU transfers.

In order to avoid these overheads, the programmer can allocate non-paged CPU memory, also known as pinned memory or pagedlocked using cudaMallocHost. However, this type of memory in the system is limited and its allocation is more expensive since it requires a user space to kernel space switch. This allows the use of fully asynchronous transfers using cudaMemcpyAsync.

Last, there is the option to allocate another type of pinned memory in the CPU side, which is also memory mapped to the GPU, using cudaHostAlloc and specifying the flag cudaHostAllocMapped. This means that no explicit copies are required between the CPU and GPU, which gives the name zero-copy. Depending on the type of the GPU, this is implemented in a different way. In a discrete GPU, ie. GPUs with their own physical DRAM memory, the copies are performed in a fined-grained manner using the DMA engines to transfer the data over the PCIe link. On the other hand, in embedded (integrated GPUs) which share the same main memory with the CPU, the GPU directly accesses the same memory as the CPU. Of course in both cases it is up to the programmer to

\footnotetext{
${ }^{1}$ CUDA also provides a feature called Unified Memory, which takes this responsibility away. Despite the increase in productivity, the performance of this feature heavily depends on the application's memory access patterns and it adds even more black-box behaviour to the memory management and its timing, which makes it less suitable for critical systems. For this reason, we do not discuss this feature in the rest of this paper.
}

ensure the consistency of the shared memory between CPU and GPU. This functionality is supported by a feature known as UVA (Unified Virtual Addressing), which allows both the CPU and the GPU to operate using the same virtual address. It is worth to note that UVA is not the same with Unified Memory, which as we explained is not appropriate for critical systems and therefore is not considered in this study. On the contrary, Unified Memory is implemented using the UVA feature.

\section{MotivationAL EXAMPLE}

Now that we have a clear idea about the different memory allocation options in CUDA, we can see a motivational example which explains the need for understanding the internals of GPU memory allocations.

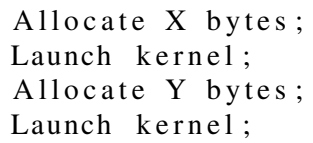

Listing 1. Motivational Example

We execute the code shown in Listing 1 on a Jetson TX2 platform, which is an embedded NVIDIA platform with an integrated GPU and we measure the execution time of the 4 GPU-related calls shown in the listing using nvprof, NVIDIA's profiler.

In Figure 1 we see the results of running the example with two allocations of the same size (1024 bytes). We notice that the first allocation takes considerable time, while the second one is shorter and the same happens on the first and second kernel launches.

However, when we allocate two chunks of memory with different sizes (1024 and 4096 bytes), we notice that always the first allocation and the first kernel launch for a given memory size take similar time (Figure 2). We notice the same trend for all the 3 different types of allocation introduced in the previous section (paged, pinned and zero-copy).

This observation indicates that the underlying memory allocator implemented in the closed source GPU runtime/driver manages each of the memory allocations of different sizes in a separate way. The question that is raised is following: can we determine the internals of this memory allocator, so that we can know the exact system memory allocated and predict which of the GPU related calls are expected to take longer? In the following sections we will introduce our methodology to discover the memory allocator internals. However, as a first step we need to examine common characteristics of memory allocators proposed in the literature for CPUs, since we suspect that the implemented memory allocator is very probable to follow a known design instead of being designed from scratch.

\section{BACKGROUND ON MEMORY ALLOCATORS}

A memory allocator provides memory to a program when requested and takes it back when the program frees it. It also keeps track of the regions of memory that have been assigned and the regions that are free to assign, using an auxiliary data structure. The main goal of an allocator is to do these tasks in the least possible amount of time minimising memory waste [8].

Initially the memory allocator reserves a contiguous chunk of memory which is used as pool, to satisfy dynamic memory requests. When the pool is full, the allocator expands by reserving a new pool. Depending on whether the allocator is implemented in the operating system or at user space, the memory for its pool is reclaimed by using a predefined range of addresses or a preallocated memory region in 


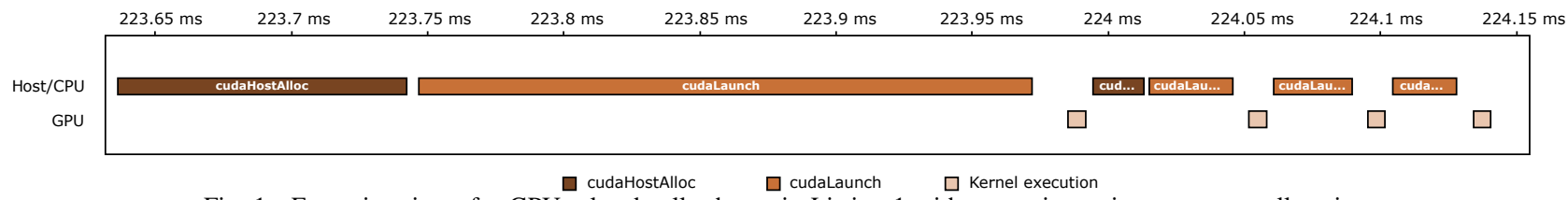

Fig. 1. Execution times for GPU related calls shown in Listing 1 with same size, using zero-copy allocations.

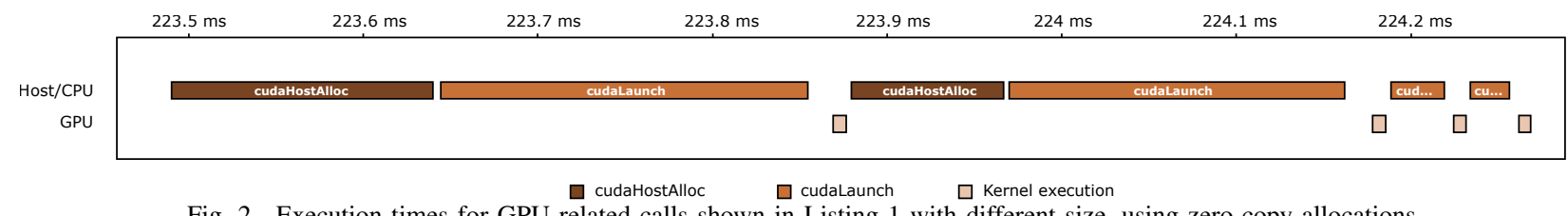

Fig. 2. Execution times for GPU related calls shown in Listing 1 with different size, using zero-copy allocations.

the former case, or using the break or mmap system calls in the latter. Custom memory allocators can also use the standard C library calls such malloc.

A common challenge for a memory allocator is that programs may free the allocated memory in any order, creating holes between used blocks. Note that for efficient representation, block sizes are usually powers of two and they have a minimum granularity. The proliferation of small holes leads to the creation of unusable blocks of memory, a problem known as fragmentation.

Fragmentation leads to memory waste, incrementing the amount of memory used by the allocator. External fragmentation occurs when the available free blocks are too small for the requested size or when the allocator is unable to split bigger blocks to satisfy smaller requests. Internal fragmentation occurs when a block larger than needed is assigned, leaving wasted memory inside the block. To avoid fragmentation, techniques like splitting free blocks (to satisfy smaller requests) and coalescing free blocks (to create larger blocks) are used in conjunction with an allocation policy.

As stated in [8], [9], [10] there are different policies and mechanisms used by memory allocators to manage memory efficiently:

Sequential fits: memory allocators in this category are based in a single linear list to manage the free blocks of memory. A best fit allocator searches the smallest free block in the list large enough to satisfy a request. A first fit allocator searches from the beginning of the list and uses the first free block large enough to satisfy the request. A next fit allocator begins the search from the last used position. A worst fit allocator looks for the largest free block in the list.

Segregated free lists: such memory allocators use an array of free lists, having one list for each block size. When a program requests memory, the allocator uses the list with the smallest block size large enough to satisfy the request. The fit of the allocations is not always perfect because the available block sizes are limited, which causes some internal fragmentation. Some segregated free lists allocators use size classes to put together a range of sizes in the same list.

Buddy systems: these allocators allocate memory in fixed block sizes which are split in two parts (or coalesced together) repeatedly to obtain blocks of the requested size. A free block can only be merged with it's buddy, so coalescing usually is fast.

Indexed fits: some memory allocators, instead of searching sequentially in a free list, use a more complex indexing data structure like a tree or a hash table to keep track of unallocated blocks. The use of this type of indexed structures leads to faster searches and allocations.

Bitmapped fits: these allocators use a bitmap to keep a record of the used areas of the heap. A bitmap is a vector of one-bit flags where each bit represents a word in the heap. The search in a bitmap is slower than in an indexed structure, however, the memory consumption is lower because it does not need to store the size of the blocks.

\section{REVERSE ENGINEERING CUDA'S MEMORY ALLOCATORS}

After reviewing the properties of existing memory allocators, we can design a methodology in order to discover the internals of the CUDA memory allocators. Note that we are interested in the key parameters of the memory allocator which affect its memory consumption and timing behaviour, but we are not after obtaining every single detail about its design ie. whether its free list is implemented using a list, tree or a bitmap, since such a task may not be entirely possible to achieve or at least not with a reasonable amount of effort. Furthermore and most importantly it does not affect resource provisioning in the same degree to the other parameters.

Without loss of generality, we focus on the same architecture we used for the motivational example. In fact, as we show in the next Section, the same methodology is applicable to all NVIDIA GPUs we tried, ranging from old to bleeding edge GPU models. Moreover, since our methodology does not depend on CUDA, it could also be applied on non-NVIDIA GPUs programmed in OpenCL.

Starting from the zero-copy allocation scenario, we want to identify the basic design of the memory allocator which is used in order to allocate pinned memory in the CUDA runtime and driver. The fact that the allocation for different sizes results in significantly longer execution times for the first allocation, means that the allocator follows a segregated free list design. Therefore, the next step is to identify its size classes as well as the pool size of each free list.

In order to achieve our goal, we design carefully crafted memory allocation experiments and observe their behaviour in order to extract the information we are after. The entire methodology is implemented using a fully automated set of scripts, that can be executed in any system featuring an NVIDIA GPU and extract its memory allocator properties. Our code is available at [11].

\begin{tabular}{ll}
\hline Algorithm 1: Pool size extraction \\
\hline Output: pool_size \\
$\mathbf{1}$ Allocate 1 byte of pinned memory \\
$\mathbf{2}$ Capture mmap system call \\
$\mathbf{3}$ Extract len argument from mmap system call \\
$\mathbf{4}$ pool_size $\leftarrow$ len \\
$\mathbf{5}$ Free memory allocated \\
\hline
\end{tabular}


Pool Size: In order to identify the pool size of each list, we first create an experiment in which we allocate the minimum amount of memory as shown in Algorithm 1. Since pinned memory has to be requested from the operating system, a user space to kernel space transition based on a system call is required. We monitor the system calls of the executing process using the strace utility, which intercepts the system calls as well as their parameters.

We notice that the memory allocation call generates a mmap system call, whose second argument corresponds to the size of the memory pool for the list. In our platform, this size is $2 \mathrm{MB}$.

As a validation, running strace on the example of Listing 1 reveals a mmap only on the first allocation of each size, both with the same size of $2 \mathrm{MB}$, which explains their longer execution time.

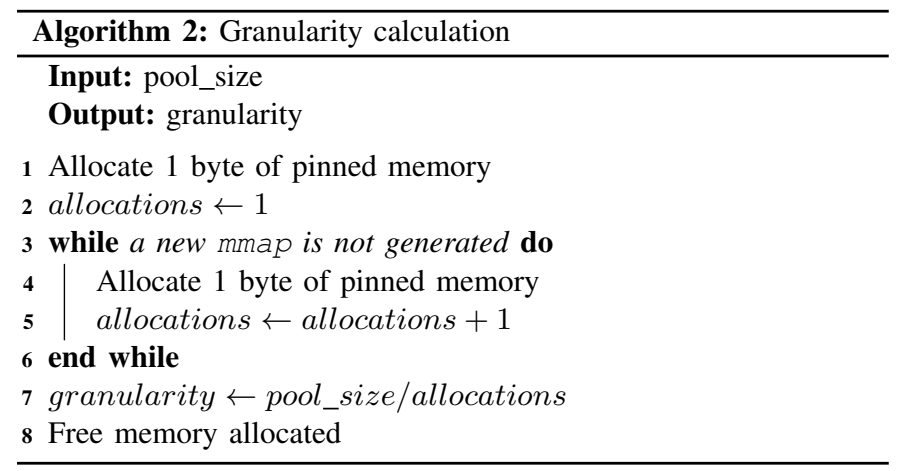

Allocation Granularity: Once we know the memory pool size, we need to identify the minimum memory size which corresponds in a single entry within the free list. We achieve this by applying Algorithm 2. The idea is simple: we try to repeatedly allocate the minimum size, until the free list is expanded, by using a new memory pool, which is indicated by a mmap call in the strace. In our platform, this happens after 4096 allocations, which means that each allocation reserved a 512 bytes entry within the free list.

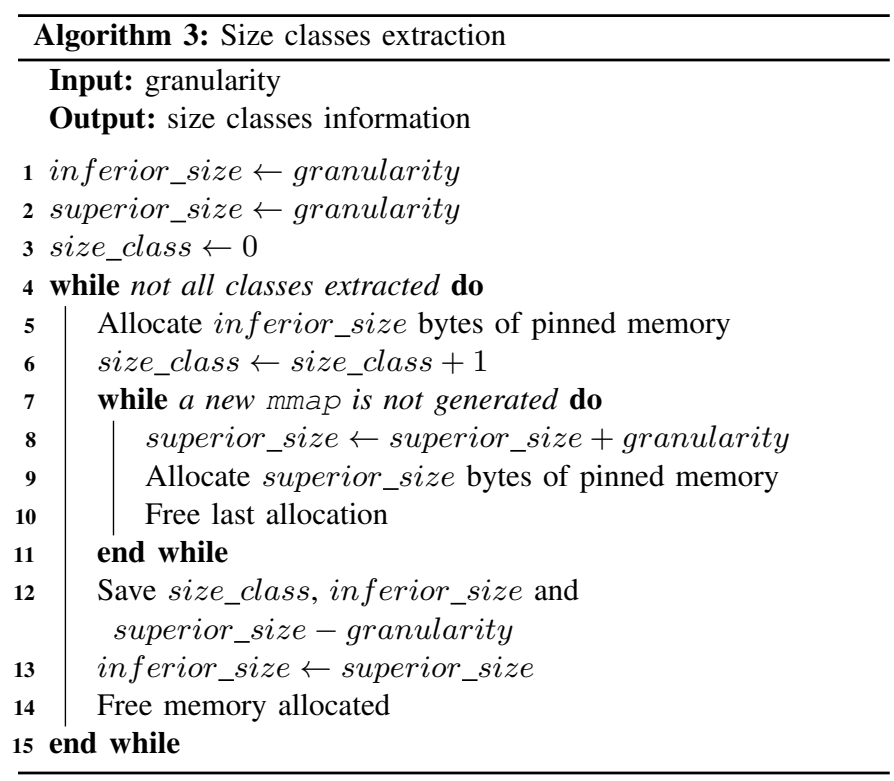

Size Classes: Knowing the size of each free list and the allocation granularity, we can focus on detecting how many free lists are kept by the allocator, each corresponding to a different size class. In Algorithm 3 we start creating allocations of increasing sizes, by using the granularity as an increment factor. If a new pool is not created (no new mmap) we free the allocation and try the next size. This way we prevent the case that the existing pool used for the current size class is expanded and therefore generating a false positive mmap.

In this experiment, we also validate that the pool size and granularity obtained for the first size class using Algorithms 1 and 2 respectively, hold also for each of the other free lists corresponding to the rest of the size classes. However, this validation is not shown in Algorithm 3 for clarity. This is achieved by using the same algorithms, but instead of allocating 1 byte, we allocate minimum size corresponding to the examined size class. We confirm that in all our experiments, these values are consistent among all the size classes for the examined systems described in the Results Section.

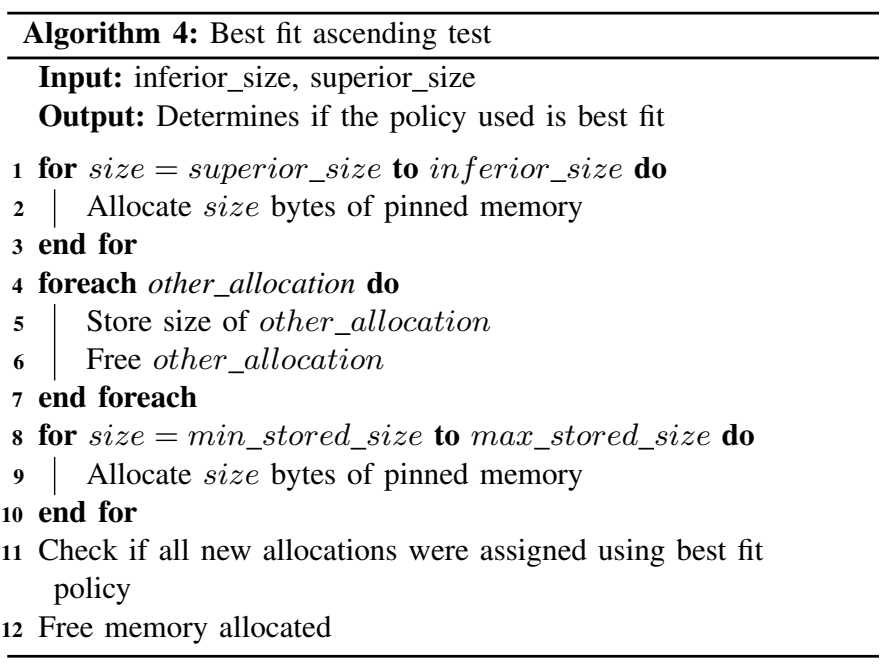

Allocation Policy: Having obtained all the parameters of the memory allocator, it only remains to identify the policy used in a free list. For this reason, we created validation tests for each type of the four main policies: first fit, best fit, next fit and worst fit. Algorithm 4 shows one these tests checking for the best fit policy. We first create a number of allocations with a decreasing size corresponding to the entire range of allowed sizes for a given size class, so that all allocations are held in the same free list (lines 1-3). Since at this point the free list is empty, each allocation takes the next available free block, resulting in consecutive allocations in the list.

Next, we start freeing every other allocation, creating free blocks of decreasing size and keeping track of their size (lines 4-7). In the final step, we start allocating the same size of blocks that were released in the previous step, but in the reverse order (lines 8-10). That is, each new allocation best fits in the last block of the free list. If the allocator follows a best fit policy, it will result in allocating the same positions as the ones that were freed in the previous step. Otherwise, eg. if the allocator follows a first fit policy, then the allocations would be suboptimal, resulting in an expansion of the original pool.

In order to perform the validation, we use multiple measures. First we use strace to validate that there is no expansion of the pool during lines 8-10. Moreover, we keep track of the addresses returned by each and make sure that the new allocations correspond to their best locations, which were their old locations.

Note that the presented example is only one of the variations of the policy validation tests, which are not shown here due to the lack of space and because they are quite similar. In particular, we have versions which perform the allocations in reverse order, or applying the last step (lines 8-10) in random order, in order to check whether 
the policy instead of best fit follows a LIFO (Last-In Last-Out, stacklike) policy. Another variation of this test uses allocations of the same size, in order to identify what is the allocation policy in the presence of multiple equal size blocks.

Coalescing: In this experiment we perform a series of allocations with arbitrary sizes which however can be rounded up to the same size in a given size class. Next, we create two neighbouring free blocks in the middle of the free list. In the following, we allocate a single block with size equal to the addition of the free blocks and we check whether the allocator merges the blocks or creates a new allocation in the free list.

Splitting: This experiment is similar to the previous one, with the difference that only one block is freed in the free list. Then a smaller size block is allocated, to check whether the allocator splits the free block, or the new allocation takes place elsewhere in the free list.

Expansion Policy: For this experiment we perform allocations for a given size class, until the pool is expanded one or multiple times. First we check whether the pool is expanded when it is full - after allocating exactly the same size of allocations with the pool size - or earlier, when an occupancy threshold in the list is exceeded. Next we free a block from the first pool, and perform a new allocation. This way we can check whether the allocation policy is applied across all the pools of the same size, or whether an alternative policy is applied eg. only to the last allocated pool.

Shrinking: Finally, we check whether the memory allocated for expanded memory pools is returned to the system. This is similar to the previous experiment. We perform allocations of the same size class until the memory pool is expanded several times and then we free all the allocations of a given memory pool. We validate whether the memory pool is returned to the system by observing a munmap after its last block is freed. Moreover we check whether only a certain memory pool is returned eg. only the last allocated or any of them.

Timing: The methodogy we presented so far corresponded to the case of pinned memory and in particular with zero-copy. In this case, in addition to the mmap during memory allocation calls, we obtain also ioctl system calls during the kernel launches. These system calls are used in order to communicate with device drivers. We observe that in the first kernel execution after a new pool created for a new size class, the kernel invocation has an extra ioct 1 call. We attribute the longer execution time of these kernels in this additional ioct 1 , which we speculate that is responsible for performing the memory mapping of the host pinned memory to the GPU's MMU (Memory Management Unit).

Paged-memory Allocator: The previously presented methodology is also appropriate without any modifications for the conventional pinned memory allocation, in which there is an one-to-one correspondence of CPU and GPU allocated memory. However, for the memory allocator used for the paged-memory allocations we need a slightly different way to observe its internals.

In particular, the paged-memory allocations do not require a userto-kernel switch and therefore its parameters cannot be obtained using strace. However, we assume that the same allocator design used for pinned memory for CUDA is also used for managed memory within CUDA, in order to reduce development and verification costs. As we comment in the Results Section, this assumption is fully validated. Since strace is not applicable in this case, the observation of the memory allocator's behaviour is applied by instrumenting the code with gdb in order to obtain the API call parameters and the returned pointers to the allocated blocks. Also, the timing behaviour is observed as previously, using NVIDIA's profiler. With these modifications, the previously presented algorithms are also used to obtain the key properties of the paged-memory allocator, too.

\section{REsults}

\section{A. Obtained Properties of CUDA allocators for various GPU models}

In this Section, we provide the results we have obtained using our methodology on a wide range of NVIDIA GPUs, ranging from very old products with capability 1.1 to the latest NVIDIA's embedded SoC Nano, as shown in Table I.

TABLE I

TESTED GPU PLATFORMS

\begin{tabular}{lcccl}
\hline \multicolumn{1}{c}{ Device Name } & $\begin{array}{c}\text { Comp. } \\
\text { Capabil. }\end{array}$ & $\begin{array}{c}\text { Runtime/ } \\
\text { Driver }\end{array}$ & $\begin{array}{c}\text { Kernel } \\
\text { Version }\end{array}$ & $\begin{array}{c}\text { GPU } \\
\text { Type }\end{array}$ \\
\hline GeForce 9300M GS & 1.1 & 6.5 & 3.19 .0 & Discrete \\
Quadro FX 3700 & 1.1 & 6.5 & 3.12 .9 & Discrete \\
GeForce GTX 960M & 5.0 & 10.0 & 4.15 .0 & Discrete \\
GeForce GTX 1050 Ti & 6.1 & 9.2 & 4.15 .0 & Discrete \\
GeForce GTX 1080 Ti & 6.1 & 9.2 & 4.15 .0 & Discrete \\
Tegra X1 (Nano) & 5.3 & 10.0 & 4.9 .140 & Integrated \\
Tegra X2 (TX2) & 6.2 & 9.0 & 4.4 .38 & Integrated \\
Xavier & 7.2 & 10.0 & 4.9 .108 & Integrated \\
\hline
\end{tabular}

We have implemented our methodology in a fully automated set of scripts performing the experiments described in the previous section. Once the scripts are executed, in a few seconds a report is generated with the information about the memory allocator. In the Listing 2 we can see the generated report about the NVIDIA's TX 2 platform, which we used in the discussion of the previous Sections.

We observe that the pool size is $2 \mathrm{MB}$ and the minimum allocation granularity is 512 bytes. The allocator is using 6 size classes, with the last one ranging up to the pool size. Larger allocations are always rounded up to the next $4 \mathrm{~KB}$ multiple, which is the system's page size. The allocator is implementing a Segregated Lists Allocator with best fit policy. In the event of expansion, the allocator is keeping a stack of pools. Deallocations can happen to any of the pools, however new allocations are only allocated in the last created pool. The allocator frees the memory used by any pool when all its blocks are freed.

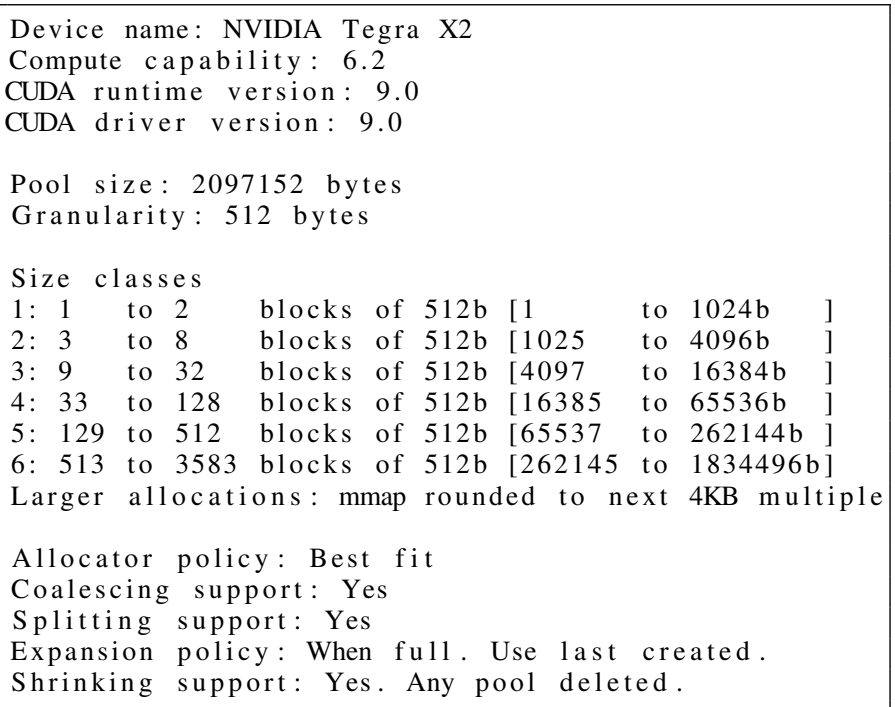

Listing 2. NVIDIA TX2 memory allocator report

Regardless of the version of the driver or the hardware, we obtained exactly the same results for the following GPUs: GeForce GTX 1080 
TABLE II

GPU MEMORY ALLOCATIONS IN EDGE DETECTION TASK (TX2)

\begin{tabular}{llr}
\hline \multicolumn{1}{c}{ Variable } & \multicolumn{1}{c}{ Type } & \multicolumn{1}{c}{ Size } \\
\hline Input Image $(640 \times 480)$ & int8 RGB & 921600 bytes \\
Filter Kernel $(3 \times 3)$ & int8 & 9 bytes \\
Output Image $(640 \times 480)$ & int8 & 307200 bytes \\
\hline Total: & & 1228809 bytes \\
\hline
\end{tabular}

TABLE III

GPU MEMORY ALLOCATOR USAGE IN EdGE DETECTION (TX2)

\begin{tabular}{llrrr}
\hline Variable & $\begin{array}{c}\text { Size } \\
\text { Class }\end{array}$ & Size & $\begin{array}{r}\text { Occupied } \\
512 \text { b Blocks }\end{array}$ & \multicolumn{1}{c}{$\begin{array}{c}\text { Occupied } \\
\text { Size }\end{array}$} \\
\hline Input Image & 6 & 921600 bytes & 1800 & 921600 bytes \\
Filter Kernel & 1 & 9 bytes & 1 & 512 bytes \\
Output Image & 6 & 307200 bytes & 600 & 307200 bytes \\
\hline Total: & & & 1229312 bytes \\
\hline
\end{tabular}

Ti, GTX $1050 \mathrm{Ti}$ and Xavier. For the GPUs GeForce GTX 960M and TX1 Nano we also obtained identical results but with the pool size being 1MB. For the older GPUs, Quadro FX 3700 and GeForce 9300M GS we obtained a pool size of $1 \mathrm{MB}$ but 256 bytes granularity.

Our results indicate that the same properties are followed by both the memory allocator for paged and pinned memory, including zero-copy. However, our system call and timing analysis for understanding the sources of variability in the execution time of GPU related calls has revealed that in the newer devices which support UVA (the ones with compute capability more than 2), only the zero-copy scenario is supported, regardless of whether the flag cudaHostAllocMapped is used.

\section{B. Exploiting the knowledge of CUDA allocators in Automotive case studies' resource provisioning}

The ultimate purpose of exposing the internals of the CUDA allocators, is this knowledge to be leveraged to compute precisely the amount of memory used by critical applications. As explained in the introduction, this will be essential when GPUs will be incorporated in avionics and automotive RTOSes. Moreover, in current general purpose operating systems it allows to make sure that the system can safely accommodate the memory and timing requirements of the application, without the use of unpredictable swap memory.

In order to demonstrate these benefits, we apply our knowledge on two automotive case studies used in modern vehicles' environment perception: a model-based generated safety-critical automotive task, implementing a sobel filter for edge detection and a pedestrian detection task [12]. The former, edge detection, is very common in both ADAS (Advanced Driving Assistance Systems) and autonomous driving for numerous tasks such as lane departure [13], sign [14] and car detection [15]. Pedestrian detection is also used for ADAS, eg. automated breaking as well as for autonomous driving.

When we execute our memory allocator properties detector on a given platform, we generate a configuration file with its properties. We have created a library exposing the CUDA memory allocation API calls, which is preloaded before a GPU program execution. This way, we can intercept all memory requests and their sizes and based on the configuration file, we can provide details about the actual memory consumption of the allocator, as we present in the results of the two case studies next.
TABLE IV

GPU MEMORY ALLOCATIONS IN PEDESTRIAN DETECTION (TX2)

\begin{tabular}{lrrr}
\hline \multicolumn{1}{c}{ Variable } & Allocs. & Individual Size & \multicolumn{1}{c}{ Total Size } \\
\hline Input Image $(640 \times 480)$ & 1 & 307200 bytes & 307200 bytes \\
Output Image $(640 \times 480)$ & 1 & 307200 bytes & 307200 bytes \\
Classifier & & & \\
Struct A & 1 & 32 bytes & 32 bytes \\
Struct B $(30 \times 16$ array) & 1 & 480 bytes & 480 bytes \\
Struct C $(250 \times 32$ array) & 30 & 8000 bytes & 240000 bytes \\
Struct D & 7500 & 84 bytes & 630000 bytes \\
\hline Total: & 7534 & & 1484912 bytes \\
\hline
\end{tabular}

1) Edge Detection: Table II shows the dynamically allocated memory, explicitly allocated in the program. We notice that the input is a 3-component (RGB) image $640 \times 480$ and a $3 \times 3$ filter kernel, while the output is a single component $640 \times 480$ image, containing the detected edges. Without knowing the internals of the CUDA memory allocator, when the task is executed on the TX2 platform with zero-copy pinned memory a system engineer might provision 1228809 bytes memory consumption.

However, Table III shows the actual memory used by the memory allocator. We notice that we have allocations from two different size classes. This means that two memory pools are created, with $2 \mathrm{MB}$ each. Each of these creations will increase the execution time of two memory allocation calls, the first ones corresponding to these class sizes, as well as the execution time of the first kernel invocation following these allocations.

Therefore, the total memory consumption to be provisioned is $4 \mathrm{MB}$ for this platform and configuration, which is $3.4 \times$ more than it was expected, due to internal fragmentation. The memory allocator though is only using a fraction of those. In the first free list, the $3 \times 3$ kernel is occupying a single block of 512 bytes instead of 9 bytes due to the minimum block granularity, while in the other free list 1228800 bytes are occupied compared to the $2 \mathrm{MB}$ of the pool, resulting in $58 \%$ free list occupancy.

On the other hand, in a Nano platform, each memory pool occupies $1 \mathrm{MB}$. However, the two images exceed the memory pool size for size class 6 , requiring the memory pool to expand. Therefore the allocator uses $3 \mathrm{MB}$ for its pools, which is $2.6 \times$ larger that the memory explicitly allocated by the application. In older GPUs like the GeForce 9300M GS, the figures are almost identical, with the difference of the block size of 256, which slightly changes the occupied size in the pool for the filter kernel.

If the application is configured to use pinned memory but not zerocopy, the above numbers are correct, too. The only difference is that in this case both CPU and GPU memory is used, which doubles the aggregate memory consumption.

Finally, if the application is configured to use paged memory, the memory consumption is also doubled because both CPU and GPU memory are used ${ }^{2}$. The difference in this case is that a pinned buffer provided by the operating system is also used for performing the

${ }^{2}$ In fact the CPU paged memory consumption in that case is closer to the explicitly allocated memory using malloc, since the GNU memory allocator [16] only uses 8 byte aligned blocks in 32-bit platforms and 16 byte aligned blocks in 64 bit ones and it does not use segregated lists. Moreover, the memory pool in CPU is lazily allocated, which means that the OS only reserves the pages of the heap which have been accessed. However, considering equal CPU and GPU memory consumption simplifies the CPU side memory analysis and provides a safe upper bound for a safety critical system in which lazy allocation is not used. 
TABLE V

GPU Memory allocator usage in Pedestrian Detection Task

\begin{tabular}{|c|c|c|c|c|c|c|}
\hline Variable & $\begin{array}{l}\text { Size } \\
\text { Class }\end{array}$ & $\begin{array}{l}\text { Individual } \\
\text { Size }\end{array}$ & $\begin{array}{c}\text { Occupied } \\
\text { 512b Blocks }\end{array}$ & $\begin{array}{c}\text { Individual Occupied } \\
\text { Size }\end{array}$ & Allocations & $\begin{array}{c}\text { Total Occupied } \\
\text { Size }\end{array}$ \\
\hline Input Image & 6 & 307200 bytes & 600 & 307200 bytes & 1 & 307200 bytes \\
\hline $\begin{array}{l}\text { Output Image } \\
\text { Classifier }\end{array}$ & 6 & 307200 bytes & 600 & 307200 bytes & 1 & 307200 bytes \\
\hline Struct A & 1 & 32 bytes & 1 & 512 bytes & 1 & 512 bytes \\
\hline Struct B & 1 & 480 bytes & 1 & 512 bytes & 1 & 512 bytes \\
\hline Struct C & 3 & 8000 bytes & 16 & 8192 bytes & 30 & 245760 bytes \\
\hline Struct D & 1 & 84 bytes & 1 & 512 bytes & 7500 & 3840000 bytes \\
\hline Total: & & & & & 7534 & 4701184 bytes \\
\hline
\end{tabular}

transfers. However, this buffer is shared among different applications and as such it does not need to be taken into account when computing the total memory consumption of the system, when multiple critical tasks are consolidated in the same platform.

2) Pedestrian Detection: This application is significantly more complex than the previous task and it is obtained from the open source implementation of the benchmark described in [12]. In addition to the input and output images, this task uses a complex dynamically allocated cascade classifier structure. This structure consists of numerous smaller dynamically allocated structures with sizes ranging from 32 bytes to 84 bytes arranged in arrays, requiring a total of 7534 dynamic memory allocations as shown in Table IV.

In a zero-copy scenario, the CPU and the GPU can use the same memory, therefore the complex structure can be used as is in the GPU, gaining in programmability.

Table IV summarises the different GPU allocations of the application. Without knowing the internals of the GPU allocator, a system engineer would provision 1484912 bytes, out of which 870512 correspond to the structure of the classifier.

However, the Table V shows the actual memory consumption within the memory allocator. Again we notice that the allocations are rounded up to 512 byte multiples, since this is the minimum allocation granularity in the allocator, which penalises small allocations. In this task there are 3 size classes used.

In platforms like the NVIDIA TX2 where the memory pool is $2 \mathrm{MB}$, a single pool is enough for class sizes 3 and 6 . However, for the class 1 the total size exceeds $2 \mathrm{MB}$, which requires the free list to expand to accommodate the total of 3841024 bytes required for this size class. Therefore the allocator uses $8 \mathrm{MB}$ in total, which is $5.6 \times$ more than the initially provisioned one.

For platforms like Nano with $1 \mathrm{MB}$ pool size, again the class sizes 3 and 6 can use a single pool, while the class 1 requires 4 pools. Therefore, the total consumption of the allocator is $6 \mathrm{MB}, 4.2 \times$ bigger than the memory explicitly requested by the application.

In the case of paged-memory or pinned memory without zero copy, the complex classifier structure cannot be used, since the pointers it contains are not valid across the different CPU and GPU address spaces. For this reason, the authors of [12] have used a single allocation for the entire structure, which is partitioned accordingly. This is similar to a custom GPU memory allocator, allowing a more predictable behaviour. In that case, a single 870512 bytes allocation is requested, which can fit in a single pool of either $2 \mathrm{MB}$ or $1 \mathrm{MB}$ depending on the device. Inside this pool, it will occupy 1701 blocks of 512 bytes, occupying 870912 bytes in the free list.

Since only a single size class is actually used in this case (class 6), the total space used inside the free list will be 1485312, so the total memory consumption of the allocator is the $2 \mathrm{MB}$ of the free list (same amount divided in 2 free lists for devices with pool size of $1 \mathrm{MB}$, like the Nano), which is $1.4 \times$ more than the initially provisioned one. Moreover, as in the previous task, since the application under this scenario requires both CPU and GPU memory, this amount is doubled.

\section{RELATED WORK}

In this Section we present some previous works in the literature similar to our work. We can categorise these works in articles related to resource allocation and reverse engineering techniques in GPUs and CPU memory allocators.

GPU Memory Allocators. Multi-core memory allocators like the one proposed by Berger et al. [17], has been shown not to scale well with many-core architectures like GPUs. For this reason, some authors have approached the GPU resource management topic by creating custom memory allocators suited for many-core architectures:

Huang et al. [18], [19] proposed XMalloc, a memory allocator based in two techniques: allocation coalescing (aggregation of memory allocation requests from SIMD-parallel threads to be handled by the CUDA allocator) and buffering of freed blocks for faster reuse using parallel queues. Results on a NVIDIA G480 GPU showed that XMalloc magnified the CUDA allocator throughput by a factor of 48 .

Steinberger et al. [20] showed that traditional memory allocation strategies used by CPUs are not suited for the use on GPUs and proposed ScatterAlloc. This allocator reduces collisions by scattering memory requests using hashing. Experimental results showed that ScatterAlloc was about 100 times faster than the CUDA allocator and up to 10 times faster than XMalloc.

Widmer et al. [21] proposed FDGMalloc, which makes use of the SIMD parallelism present in GPUs to significantly speed-up the allocation of dynamic memory. The authors compared their implementation with the CUDA allocator and with ScatterAlloc, achieving a speed-up of several orders of magnitude.

A common characteristic in all these works is that they focus their analysis in comparing the performance of their allocators with the performance of the CUDA allocator, without trying to understand its internal structure or the way it works as we do in this paper. Moreover, these works obtain their memory through the CUDA memory allocator, so they are still susceptible to the timing effects of its usage.

Reverse Engineering Works on GPUs. The black box nature of the GPUs has lead to the creation of some research works oriented to the use of reverse engineering techniques to get information about their internal characteristics.

Wong et al. [22] developed a microbenchmark suite to measure various undisclosed characteristics of the processing elements and memory hierarchies of a NVIDIA GTX280 GPU. Their results 
validated some of the hardware characteristics publicly available and revealed some other undocumented hardware structures used for control flow and caching. Following a similar approach, Mei et al. [23] exposed previously unknown characteristics about the memory hierarchy of Fermi, Kepler and Maxwell NVIDIA GPUs.

Amert et al. [24] applied black-box experimentation to a NVIDIA TX 2 GPU. Based on results, they defined a set of rules describing the behaviour of the NVIDIA TX2 scheduler. The same group later extended their work on software and disclosed a set of non-obvious pitfalls to avoid when using CUDA-enabled GPUs for safety-critical systems [25].

All these works are based in applying reverse engineering techniques to hardware or software of GPUs, however, none of them is oriented to get information about the memory allocation system and leverage it, which is the focus of our study.

Reverse Engineering Memory Allocators. Eventhough memory allocation is an extensively researched area, the only work to our knowledge related to reverse engineering memory allocators is the MemBrush tool, proposed by Chen et al. [26]. The purpose of MemBrush is to detect the API functions of custom memory allocators in stripped binaries. MemBrush has been used to improve other reverse engineering tools like Howard [27], which is used to extract data structures from $\mathrm{C}$ binaries without having any symbol tables.

To the best of our knowledge, our paper is the first work oriented to extract information (real memory usage, size classes and allocation policy) about a closed source GPU memory allocator and to analyze the benefits of this information for critical systems.

\section{CONCLUSIONS}

In this paper we presented a methodology and an automated way to extract information about the internals of the CUDA memory allocators. We applied our method in a wide range of GPUs and we identified that there is only a slight difference between different GPUs, in the amount of memory used internally as a pool and the granularity, in particular in older GPUs.

Moreover, we have applied our extracted information about the memory allocator in two safety critical automotive case studies, showing how a system engineer can be benefited by this information, in order to provision the correct amount of memory. In particular we have shown that the actual memory consumption of the memory allocator can be up to an order of magnitude higher than the amount requested by the application.

\section{ACKNOWLEDGMENTS}

This work has been partially supported by the Spanish Ministry of Science and Innovation under grant TIN2015-65316-P, the HiPEAC Network of Excellence and the European Research Council (ERC) under the European Union's Horizon 2020 Research and Innovation programme (grant agreement No. 772773). Leonidas Kosmidis is also funded by the Spanish Ministry of Economy and Competitiveness (MINECO) under a Juan de la Cierva Formación postdoctoral fellowship (FJCI-2017-34095).

\section{REFERENCES}

[1] L. Kosmidis, C. Maxim, V. Jegu, F. Vatrinet, and F. J. Cazorla, "Industrial Experiences with Resource Management under Software Randomization in ARINC653 Avionics Environments," in IEEE/ACM International Conference on Computer-Aided Design, Digest of Technical Papers, ICCAD, 2018

[2] ARINC, "Avionics Application Software Standard Interface: ARINC Specification 653P1-3. Aeronautical Radio," 2010.

[3] AUTOSAR, "AUTOSAR," accessed April 2019. [Online]. Available: https://www.autosar.org
[4] Green Hills Software, "Integrity RTOS," 1996, accessed April 2019. [Online]. Available: https://www.ghs.com/products/rtos/integrity.html

[5] NVIDIA Corporation, "Self Driving Cars," accessed April 2019. [Online]. Available: https://www.nvidia.com/en-us/self-driving-cars

[6] X. Yu, H. Wang, W. . Feng, H. Gong, and G. Cao, "GPU-Based Iterative Medical CT Image Reconstructions," Journal of Signal Processing Systems, vol. 91, no. 3-4, pp. 321-338, 2019.

[7] R. L. Davidson and C. P. Bridges, "Error Resilient GPU Accelerated Image Processing for Space Applications," IEEE Transactions on Parallel and Distributed Systems, vol. 29, no. 9, pp. 1990-2003, 2018.

[8] P. R. Wilson, M. S. Johnstone, M. Neely, and D. Boles, Dynamic Storage Allocation: A Survey and Critical Review, ser. Lecture Notes in Computer Science (including subseries Lecture Notes in Artificial Intelligence and Lecture Notes in Bioinformatics), 1995, vol. 986.

[9] Y. Hasan and J. M. Chang, "A Tunable Hybrid Memory Allocator," Journal of Systems and Software, vol. 79, no. 8, pp. 1051-1063, 2006.

[10] V. Shah and A. Shah, Proposed Memory Allocation Algorithm for NUMA-Based Soft Real-Time Operating System, ser. Advances in Intelligent Systems and Computing, 2019, vol. 814.

[11] A. J. Calderón, L. Kosmidis, C. F. Nicolás, F. J. Cazorla, and P. Onaindia, "CUDA Memory Allocator Inspector." [Online]. Available: https://github.com/ajcalderont/cmai

[12] M. M. Trompouki, L. Kosmidis, and N. Navarro, "An Open Benchmark Implementation for Multi-CPU Multi-GPU Pedestrian Detection in Automotive Systems," in IEEE/ACM International Conference on Computer-Aided Design, Digest of Technical Papers, ICCAD, vol. 2017November, 2017, pp. 305-312.

[13] U. Ozgunalp, "Combination of the Symmetrical Local Threshold and the Sobel Edge Detector for Lane Feature Extraction," in Proceedings 9th International Conference on Computational Intelligence and Communication Networks, CICN 2017, vol. 2018-January, 2018, pp. 24-28.

[14] H. Vishwanathan, D. L. Peters, and J. Z. Zhang, "Traffic Sign Recognition in Autonomous Vehicles Using Edge Detection," in ASME 2017 Dynamic Systems and Control Conference, DSCC 2017, vol. 1, 2017.

[15] R. Younis and N. Bastaki, "Accelerated Fog Removal from Real Images for Car Detection," in 2017 9th IEEE-GCC Conference and Exhibition, GCCCE 2017, 2018.

[16] Free Software Foundation, "The GNU Allocator," 2019, accessed April 2019. [Online]. Available: https://www.gnu.org/software/libc/manual/html_node/The-GNUAllocator.html

[17] E. D. Berger, K. S. McKinley, R. D. Blumofe, and P. R. Wilson, "Hoard: A Scalable Memory Allocator for Multithreaded Applications," in International Conference on Architectural Support for Programming Languages and Operating Systems - ASPLOS, 2000, pp. 117-128.

[18] X. Huang, C. I. Rodrigues, S. Jones, I. Buck, and W. Hwu, "XMalloc: A Scalable Lock-Free Dynamic Memory Allocator for Many-Core Machines," in Proceedings - 10th IEEE International Conference on Computer and Information Technology, CIT-2010, 7th IEEE International Conference on Embedded Software and Systems, ICESS-2010, ScalCom-2010, 2010, pp. 1134-1139.

[19] X. Huang, C. I. Rodrigues, S. Jones, I. Buck, and W.-m. Hwu, "Scalable SIMD-Parallel Memory Allocation for Many-Core Machines," Journal of Supercomputing, vol. 64, no. 3, pp. 1008-1020, 2013.

[20] M. Steinberger, M. Kenzel, B. Kainz, and D. Schmalstieg, "ScatterAlloc: Massively Parallel Dynamic Memory Allocation for the GPU," in 2012 Innovative Parallel Computing, InPar 2012, 2012.

[21] S. Widmer, D. Wodniok, N. Weber, and M. Goesele, "Fast Dynamic Memory Allocator for Massively Parallel Architectures," in ACM International Conference Proceeding Series, 2013, pp. 120-126.

[22] H. Wong, M. . Papadopoulou, M. Sadooghi-Alvandi, and A. Moshovos, "Demystifying GPU Microarchitecture through Microbenchmarking," in ISPASS 2010 - IEEE International Symposium on Performance Analysis of Systems and Software, 2010, pp. 235-246.

[23] X. Mei and X. Chu, "Dissecting GPU Memory Hierarchy through Microbenchmarking," IEEE Transactions on Parallel and Distributed Systems, vol. 28, no. 1, pp. 72-86, 2017.

[24] T. Amert, N. Otterness, M. Yang, J. H. Anderson, and F. Donelson Smith, "GPU Scheduling on the NVIDIA TX2: Hidden Details Revealed," in Proceedings - Real-Time Systems Symposium, vol. 2018-January, 2018.

[25] M. Yang, N. Otterness, T. Amert, J. Bakita, J. H. Anderson, and F. D. Smith, "Avoiding Pitfalls when Using NVIDIA GPUs for Real-Time Tasks in Autonomous Systems," in Leibniz International Proceedings in Informatics, LIPIcs, vol. 106, 2018. 
[26] X. Chen, A. Slowinska, and H. Bos, "Who Allocated My Memory? Detecting Custom Memory Allocators in C Binaries," in Proceedings Working Conference on Reverse Engineering, WCRE, 2013, pp. 22-31.

[27] A. Slowinska, T. Stancescu, and H. Bos, "Howard: A Dynamic Excavator for Reverse Engineering Data Structures," Proceedings of the 18th Annual Network and Distributed System Security Symposium (NDSS'11), 2011. 